\title{
Waveguide characteristics of elastic pipes filled with multiphase fluids
}

\author{
Dipl.-Ing. Sebastian Wöckel, Dipl.-Ing. Hendrik Arndt, Dr.-Ing. Ulrike Steinmann, Prof. Dr. Jörg Auge \\ Institut für Automation und Kommunikation (ifak) e.V. Magdeburg, Department Measurement Techno- \\ logy and Power Electronics, Werner-Heisenberg-Str. 1, 39106 Magdeburg, Germany \\ sebastian.woeckel@ifak.eu
}

\begin{abstract}
:
This paper addresses the multimodal acoustic wave propagation in elastic pipes which can carry multiphase fluids. The waveguide characteristics of circular, fluid filled pipes and the influence of elasticity of the outer boundaries (pipe wall) in relation to the compressibility of the filling liquid and the inner boundaries due to an additional fluid phase are discussed - in comparison to the assumption of rigid walls. The presented studies base on simulations which combine the fluid pressure acoustics and the solid structure mechanics of a circular pipe which is filled with up to two fluid phases.
\end{abstract}

Key words: guided acoustic wave, pipe acoustic, dispersion.

\section{Motivation}

The industrial process monitoring with acoustic waves is a common and interesting technique especially when a non-invasive clamp-on configuration can be realised, because of its nondestructive nature and easy handling. Often the vessels where the acoustic measurement is applied are of limited volume and have restricted boundaries. General bulk wave acoustics do not comply here. The tasks in process industry are technologies to monitor filling level, flow, concentration, density, particle content or distribution of multiphase media.

Since the acoustic sensor is mounted on the outer surface (e.g. pipe wall), reflections and acoustic mode conversions may occur. The vessel acts as a complex waveguide depending on the measurement frequency, the particular filling medium and its geometry [1], [2], [3]. The outcome of this is a dispersive axial wave velocity along the pipe wall (structure-borne waves) and even within the fluid. Since the waveguide behaviour is unknown, the dependency of the velocity on frequency (dispersion) and geometry will lead to uncertainty of the correlation of the pressure amplitude and the round trip time to changes in flow speed or variations of the properties of the bulk material, e.g. due to temperature. Further, the different coexisting dispersive high-order wave modes will cause a complex signal, whose shape and characteristics change with propagation distance.
Since a pipe is a closed system no free wave can be assumed. According to several authors the propagation of an acoustic wave within a simple cylindrical pipe depends on the mechanical impedance or elasticity of the boundary (pipe wall) for "low" frequencies [4], [5]. For "higher" frequencies the pipe acts as a waveguide and additional wave modes will occur depending on the geometry [6], [7], [8]. In particular the decrease of the sound velocity due to the elastic properties of the pipe wall was first postulated by [9] and later verified amongst others by [10]. The labels "low" and "high" are related to the acoustic cut off frequency $f_{\mathrm{g}}$ (first radial frequency) of the guided waves.

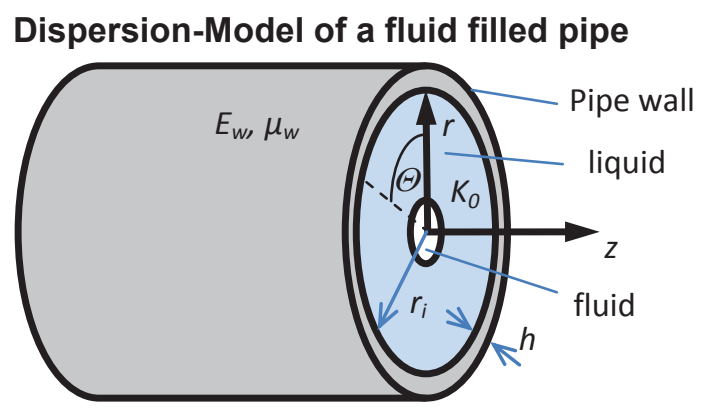

Fig. 1. Waveguide-model of fluid-filled cylindrical pipe segment with $r$ - radial component, $z$ - axial component, $K$ - compressibility, $E$ - elasticity and $\mu$-poissons ratio.

To consider the axial wave propagation inside a fluid filled pipe a simple model with axial symmetry in cylindrical coordinates and infinite periodical dimensions in in z-direction is used (Fig. 1 ). Due to this symmetry only longitudinal wave 
modes are discussed. The flexural and torsional modes are excluded by the solution. According to this simplification the pipe forms a waveguide where - besides the dominant mode - higher order wave modes may occur depending on the ratio of frequency (wavelength) to geometric dimension $\left(r_{\mathrm{i}} / \lambda\right)$. Normally those modes are dispersive and their propagation velocities depend on the frequency.

In general the wave propagation can be described with a wave equation. Equation (1) represents the wave equation for the displacement $u$ of a wave travelling along the pipe:

$$
\begin{gathered}
u(r, \theta, z, t)=\left[A \sin \left(k_{\theta} \theta\right)+B \sin \left(k_{\theta} \theta\right)\right] J_{n}\left(k_{c} r\right) e^{i\left(k_{z} z-\omega t\right)} \\
k_{c}^{2}=k^{2}-k_{z}^{2} \\
\omega=k \cdot c,
\end{gathered}
$$

Herein $A$ and $B$ are constants, $k$ the wavenumbers for each coordinate and $J_{n}$ the Besselfunction. For each layer of the pipe the amplitudes $A$ and $B$ of the partial waves must be solved for every coordinate $(r, \Theta, z)$. So, six equations need to be solved for each layer respecting the boundary conditions between the layers [7], [11].

Typically, the partial differential equations have to be solved numerically [7]. In the current work the following software tools were used:

- $\quad$ Lamb Toolbox for Matlab [12],

- $\quad$ GUIGUW (Matlab) [13] and

- Comsol Multiphysics.

The solutions of the wave equations are three different wave modes: Longitudinal-, Torsional- and Flexural- modes with an unlimited number of single modes with different cut-off frequencies, phase velocities $c_{\text {ph }}$ and damping. Longitudinal waves have a dominant displacement in radial $(r)$ and axial $(z)$ direction (see Fig. 1). In contrast torsional waves only have a displacement in $\Theta$-direction. Both modes are axisymmetric, which means that the displacement on a certain distance to the pipes center is constant along the circumference. The flexural modes have no axial symmetry and a negligible displacement in this direction. In the current work the different modes are discriminated and classified with the index $(n, m)$ [14]. The circumferential index $n=0$ classifies modes with axial symmetry and higher modes $n=1,2,3, \ldots$ represent non-symmetric fields:

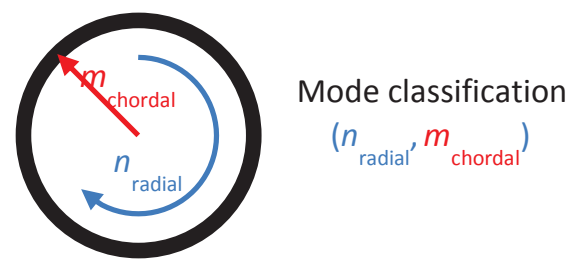

a) Longitudinal - axial symmetry

$$
\begin{aligned}
& L(0, m) m=1,2,3, \ldots \\
& U_{Z}, U_{R} \neq 0, U_{\Theta}=0
\end{aligned}
$$

b) Torsional - axial symmetry

$$
\begin{aligned}
& T(0, m) m=1,2,3, \ldots \\
& U_{Z}, U_{R}=0, U_{\Theta} \neq 0
\end{aligned}
$$

c) Flexural - no axial symmetry

$$
\begin{aligned}
& \boldsymbol{F}(\boldsymbol{n}, \boldsymbol{m}) n=1,2,3, \ldots m=1,2,3, \ldots \\
& U_{z}, U_{R}, U_{\Theta} \neq 0
\end{aligned}
$$

The index $m$ discriminates ascending modes of one class. The variable $U_{i}$ labels the effective displacement for each coordinate.

All guided $L-, \quad T$ - and $F$ - waves' phase and group velocities depend on frequency. Normally this dependency is depicted within a dispersion diagram.

Model of Superposition: To comprehend the dispersive characteristics of complex systems their dispersion diagram can be approximated by the superposition of simplified geometries (Fig. 2). The wave propagation in cylindrical multiphase systems is such a superposition of homogeneous single components (like cylinder) with isotropic materials. As a result the fluid filled pipe can be dismantled in an empty pipe with free boundaries and a water column with rigid boundaries.

The modes (dispersion curves) of the single components either can be asymptotes or direct modes of the complex system. Besides, a mode jumping will occur at mode crossing points.

Concerning the boundaries the cases free, rigid and elastic have to be distinguished. Hereby, every filling of the pipe defines the mechanical load at the inner boundary of the pipe wall, which will lead to a clear change of the dispersions characteristics of the guided wave modes
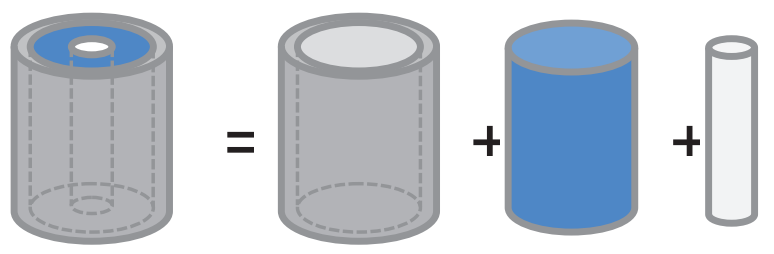

Fig. 2. Scheme for superposition of a fluid-filled cylindrical pipe. 


\section{Results}

Dispersion diagram of an empty cylindrical pipe with free boundaries: For an ideal pipe surrounded by vacuum (Fig. 3) or a fluid with low mechanical loading three fundamental dominant acoustic modes exist $[L(0,1), F(1,1)$, $T(0,1)$ ] below the first cut-off frequency (first radial frequency, $f_{\mathrm{g}}=5 \mathrm{kHz}$ for a plastic pipe corresponding to Fig. 3 and 5 [7]. Their cut-off frequencies depend on material and size of the pipe.

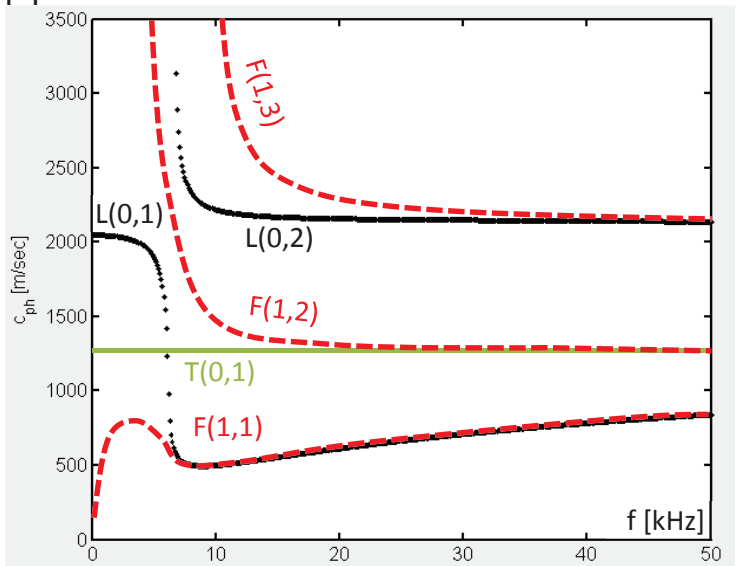

Fig. 3. Longitudinal (black), Torsional (green) and Flexural modes (dashed red) of an empty plastic pipe $\left(E=5.787 \mathrm{GPa}, \mu=0.3, \rho=1380 \mathrm{~kg} / \mathrm{m}^{3}, d_{i}=99 \mathrm{~mm}\right.$, $d_{a}=110 \mathrm{~mm}$ ) - calculated with GUIGUW
Dispersion diagram of a liquid filled pipe: In the lower frequency range the filling of a pipe leads to a clear change of the longitudinal modes (Fig. 4). The former $L(0,2)$-mode (refer Fig. 3, now dashed line in Fig. 4) splits into a variety of new L-modes. According to the model of superposition the original modes of the empty pipes (without mechanical loading) $L(0,1)$, $L(0,2)$, describe the asymptotic behavior of the fluid filled pipe. Additional asymptotes are built by the guided waves of the water column with ideal rigid boundaries. Due to the presence of multiple asymptotes the higher order modes $(m$ $>1$ ) are "jumping" between them. This results in high and low dispersive frequency intervals of each new dispersion curve (e.g. $L(0,2))$.

Within the fluid an additional axisymmetric mode M1 (Fig. 4) is formed which is correlated to the sound velocity of the fluid. For "low" frequencies its displacement is concentrated to the inner pipe [15] and therefore it is propagable by excitations sole to the fluid. Both the $L(0,1)$-mode and the $M 1$-mode have a dominant axial displacement, the first mainly within the pipe wall and the second within the fluid. By loadings on the outer pipe wall (e.g. embedding or buried pipes) two more axisymmetric modes can occur - depending on the elastic properties [8], [11]. However, the present studies concentrate on the fluid-mode $\mathbf{M 1}$. The outer pipe wall is assumed to be free.

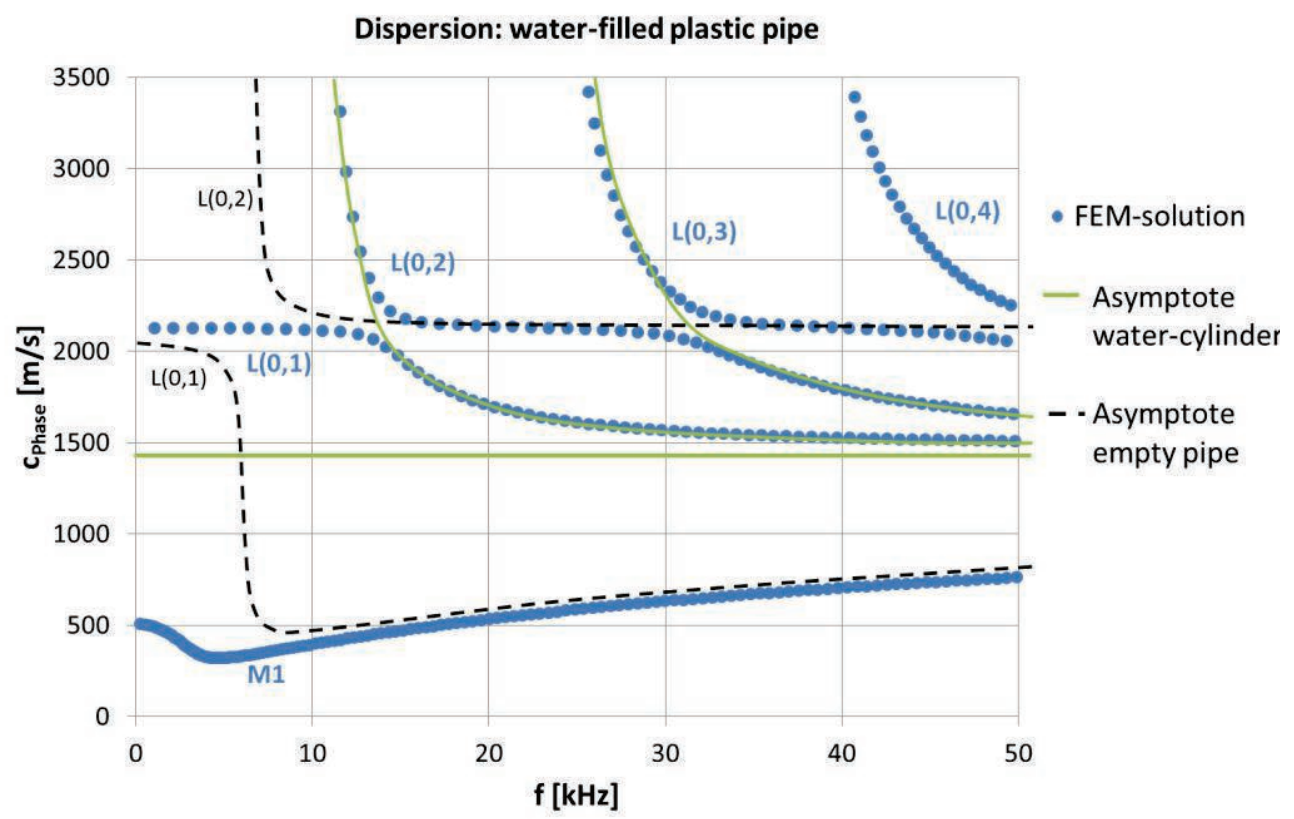

Fig. 4: 2D-FEM-solution: dispersion diagram of longitudinal modes $L$ and the fluid mode $M 1$ of a water-filled plastic pipe $\left(E=5.787 \mathrm{GPa}, \mu=0.3, \rho=1380 \mathrm{~kg} / \mathrm{m}^{3}, d_{i}=99 \mathrm{~mm}, d_{a}=110 \mathrm{~mm}\right.$ ) with identification of the asymptotes of the empty pipe (black - dashed) and water cylinder (green - solid). 


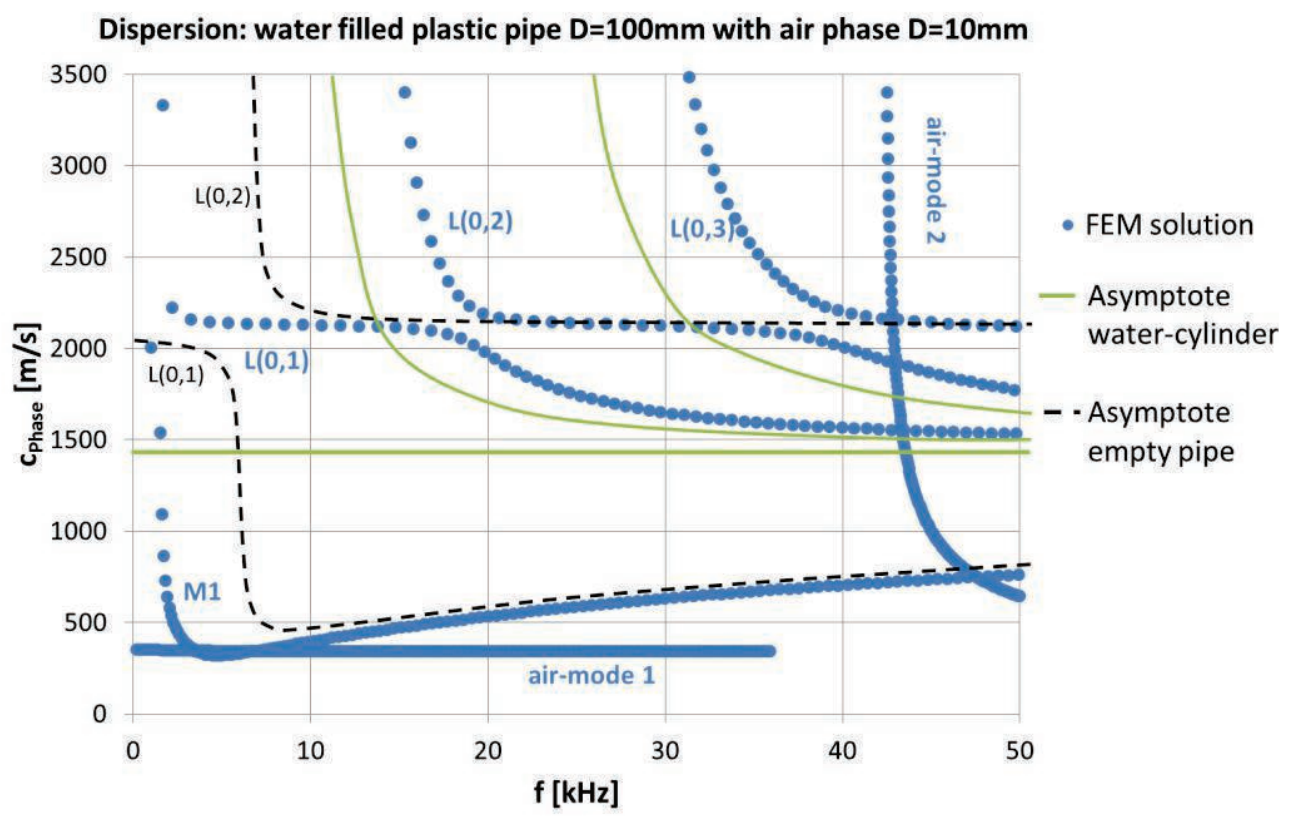

Fig. 5: 2D-FEM-solution: dispersion diagram of longitudinal modes $L$ and the fluid mode $M 1$ of a water-filled plastic pipe ( $E=5.787 \mathrm{GPa}, \mu=0.3, \rho=1380 \mathrm{~kg} / \mathrm{m}^{3}, d_{i}=99 \mathrm{~mm}, d_{a}=110 \mathrm{~mm}$ ) with inner gas phase (air $d_{\text {air }}=10$ $\mathrm{mm}$ ); with identification of the asymptotes of the empty pipe (black - dashed) and water cylinder (green - solid).

As a consequence of the liquid filling the $L(0,2)$-mode is switched to a higher cut-off frequency (Fig. 4). Below the first cut-off frequency $(10 \mathrm{kHz}$ in Fig. 4) the new fundamental $L(0,1)$-mode on the pipe wall is non-dispersive and almost constant. Furthermore the fluid mode M1:

- propagates with a velocity clearly below the velocity of a free wave $\left(c_{0}=\right.$ $1480 \mathrm{~m} / \mathrm{s}$ ),

- shows a minimum in the proximity of the first radial frequency $\left(f_{\mathrm{g}}=5 \mathrm{kHz}\right.$, empty pipe with free boundaries) and

- follows the dispersion curve of the $L(0,1)$-mode of the empty pipe (black dashed line in Fig. 4) above the radial frequency $\left(f>f_{\mathrm{g}}\right)$.

Assuming a further additional fluid phase with lower compressibility (e.g. steam or air in the center of the pipe) the dispersion diagram will slightly change for the higher frequencies. This complies with the model that the complex acoustic waveguide of a liquid filled pipe can be approximated by the superposition of cylindrical structures representing the different phases with rigid boundaries. But in the lower frequency range (below the first radial frequency $f<5 \mathrm{kHz}$ ) the fluid-mode M1 "jumps" to the former $L(0,1)$-mode of the empty pipe (compare Fig. 4. and Fig. 5).

The gas phase delivers an opposing unsymmetrical boundary condition because of the differences in the bulk modulo (water - gas / water - pipe wall). This lack in symmetry leads to the "jumping" of the M1-mode. In contrast a material which brings symmetric boundary conditions (like rigid walls or higher elasticity) would not evoke a mode-jumping.

The higher the frequency becomes the lower the influence of the gas phase (in the pipes center) gets. The acoustic displacement concentrates along the area of the inner pipe wall. This behavior corresponds to the model, so that the range of influence of a boundary (with varying impedance) is mainly limited to the wavelength of the acoustic signal. Accordingly, the fluid mode $M 1$ follows the $L(0,1)$ mode if the wavelength becomes smaller than the inner radius of the pipe (Fig. 4 and 5).

\section{Analytic Approximation}

In addition to the numerical studies three analytic solutions (2), (3) and (4) can be found in literature [4], [5], which can be used to approximate the characteristics of the fluid mode M1 - without complex simulations.

The main approximations of Kuhl [4] and Pinnington [5] base on investigations of Korteweg (1878) [10], Résal, [16] und Moens. A more exact - but also complex - solution is presented by Muggleton [17].

Both approximations represent asymptotes for the phase velocity. The approximation of Pinnington (4) is related to frequencies below the first radial frequency (2) and Kuhl's formula (3) approximates the velocity for higher frequency above the radial frequency. The first radial frequency can be calculated with (2). 


$$
f_{\text {Ring }}=\frac{c_{L}^{s 0}}{2 \pi r} \text { mit } \quad c_{L}^{s 0}=\sqrt{\frac{E_{W}}{\rho_{W}\left(1-\mu^{2}\right)}}
$$

Equ. 2. First radial frequency, $r$ - mean radius of the pipe, $\rho_{w}$-density of the pipe wall, $\mu$ - poissons ratio

$$
\frac{c^{\prime}}{c_{0}}=\frac{1}{\sqrt{1+\frac{2 K_{0}\left(d_{a} / d_{i}\right)^{2}+1}{E_{W}\left(d_{a} / d_{i}\right)^{2}-1}}}
$$

Equ. 3. Approximation of Kuhl [4], c'-sound velocity of the fluid inside the pipe; $c_{0}$ - bulk velocity of the fluid in free space; $K_{0}$ - volume elasticity of the fluid; $E_{W}$ - Elasticity of the pipe wall material; $d_{a}$ - pipes outer radius; $d_{i}$ - pipes inner radius

$$
\frac{c^{\prime}}{c_{0}}=\frac{1}{\sqrt{1+\left(\frac{d_{i}}{h}\right) \cdot \frac{K_{0}}{E_{W}}}}
$$

Equ. 4. Approximation of [5], [10], di pipes inner diameter, $h$ - wall thickness

All three equations imply that the propagation characteristics mainly depend on the effective elasticity of the pipe wall - as a combination of bulk elasticity and mechanical behavior of the whole geometry. From Equ. 3 and 4 it can be inferred that, even for infinite pipe wall thickness, the effective velocity in the fluid $c$ converges to a limit value, which depends on the elasticity only. As an example the value for steel will not cross $98 \%$ of the velocity of the free wave in water (here $c_{0}=1480 \mathrm{~m} / \mathrm{s}$ ).

\section{Verification}

The presented investigations mainly consider the dependency of the fluid-wave propagation inside a pipe on the elasticity of the pipe wall and its geometry. Referring to this two simulation based examples were demonstrated (Fig. 4 and 5).

In consecutive steps selected numerical results are validated with acoustic measurements using a water filled plastic pipe. In addition, the common low frequency approximations [4], [5] for large scale pipes with elastic boundaries are related to the numerical results and their validity is shown.

Those verifications as well as a further parameter study concerning the influences of the pipe walls elasticity, of the diameter and of the wall thickness will be presented at the conference.

\section{References}

[1] V.A. del Grosso, Analysis of multimode acoustic propagation in liquid cylinders with realistic boundary conditions, Acustica, 24:299, (1971)

[2] D.J. Korteweg, Über die Fortpflanzungsgeschwindigkeit des Schalls in elastischen Röhren, Ann. Physik. Chem., 5, 525-533 (1878)

[3] L.D. Lafleur, F.D. Shields, Low-Frequency propagation modes in a liquid-filled elastic tube waveguide, JASA, 97, 1435-1445 (1995), doi: 10.1121/1.412981

[4] W. Kuhl, Die Eigenschaften wassergefüllter Rohre für widerstands- und Schallgeschwindigkeitsmessungen, Acustica, 3, 111-123 (1953)

[5] R.J. Pinnington, A.R. Briscoe, Externally applied sensor for axisymmetric waves in a fluid filled pipe, Journal of Sound and Vibration, 173(4), 503-516 (1994), doi: 10.1006/jsvi.1994.1243

[6] L.D. Lafleur, F.D. Shields, Low-Frequency propagation modes in a liquid-filled clastic tube waveguide, in: Journal of the Acoustical Society of America, 97:1435, 1995

[7] D.C. Gazis, "Three dimensional investigation of the propagation of waves in hollow circular cylinders", 1959

[8] M.J.S. Lowe, „Matrix techniques for modelling ultrasonic waves in multilayered media, 1995

[9] H. Helmholtz, "Berichte übder die theoretische Akustik betreffenden Arbeiten", 1848

[10] D.J. Korteweg, „Über die Fortpflanzungsgeschwindigkeit des Schalls in elastischen Röhren“, 1878

[11] Long, R.; Cawley, P.; Lowe, M. (2003): Acoustic wave propagation in buried iron water pipes. In: Proceedings of the Royal Society A: Mathematical, Physical and Engineering Sciences 459 (2039), S. 2749-2770. DOI: 10.1098/rspa.2003.1148

[12] Prego-Borges, J.L., "Lamb: a simulation tool for air-coupled Lamb wave based ultrasonic NDE systems", In Proceedings of the 1st. Barcelona Forum on Ph.D. Research in Electronic Engineering (2010) Universitat Politecnica de Catalunya.

[13] Bocchini, P., Marzani, A., and Viola, E. (2011). "Graphical User Interface for Guided Acoustic Waves." J. Comput. Civ. Eng., 25(3), 202-210.

[14] M.G.Silk, "The propagation in metal tubing of ultrasonic wave modes equivalent to Lamb waves, 1979

[15] P. Morse, „Some aspects of the theory of room acoustics", 1939

[16] H. Résal, "Sur les petits mouvements d'un fluide incompressible dans un tuyau élastique", 1876

[17] J.M. Muggleton, "Wavenumber prediction of waves in buried pipes for water leak detection", 2002 